\title{
Thyroid Peroxidase Antibodies in Non-Autoimmune Hyperthyroidism Treated with Radioactive lodine
}

Authors

Affiliations

\author{
M. H. Listewnik ${ }^{1}$, M. J. Listewnik², T. Miazgowski ${ }^{3}$, K. Jaskólska ${ }^{1}$, K. Jasiakiewicz ${ }^{1}$, B. Birkenfeld
}

${ }^{1}$ Department of Nuclear Medicine, Pomeranian Medical University, Szczecin, Poland

${ }^{2}$ Department of Cardiac Surgery, Pomeranian Medical University, Szczecin, Poland

${ }^{3}$ Department of Hypertension and Internal Medicine, Pomeranian Medical University, Szczecin, Poland
Key words

- non-autoimmune hyperthyroidism

- radioactive iodine therapy

- thyroid peroxidase antibody

- hypothyroidism received $\quad$ 15.04.2016

first decision 12.07.2016 accepted 20.07.2016

\section{Bibliography}

Dol http://dx.doi.org/

10.1055/s-0042-113124

Exp Clin Endocrinol Diabetes

2016; 124: 572-576

(C) J. A. Barth Verlag in Georg Thieme Verlag KG

Stuttgart · New York

ISSN 0947-7349

\section{Correspondence}

\section{T. Miazgowski}

Department of Hypertension \& Internal Medicine

Pomeranian Medical University

Unii Lubelskiej 1

71-252 Szczecin

Tel.: + 48/91/4253550

Fax: + 48/91/425 3552

miazgowski@interia.pl

\section{Abstract \\ $\nabla$}

Introduction: Earlier studies suggest increased serum levels of thyroid peroxidase antibodies (TPOAb) in some cases with non-autoimmune hyperthyroidism. The aim of the study was to assess the incidence of hypothyroidism in patients with nodular toxic goitre and toxic adenoma at 12 months after radioactive iodine therapy in the relation to TPOAb levels.

Patients \& Measurements: The study comprised 100 patients (83 females; 17 males) treated with radioactive iodine therapy. Serum concentrations of thyrotropin, free thyroxin, TPOAb, and anti-TSH receptor antibodies were assessed at baseline and 12 months after radioactive iodine therapy.

Results: High TPOAb level ( $>60.0 \mathrm{IU} / \mathrm{mL}$ ) was found in $27 \%$ of patients at baseline and $32 \%$

\section{Introduction}

$\nabla$

Distinguishing between autoimmune and nonautoimmune hyperthyroidism (NIH) is crucial for radioactive iodine therapy (RIT) because different approaches to patient preparation and follow-up are required $[1,2]$. In a typical course of NIH the medical history, thyroid hormone levels, ultrasonographic appearance, and the thyroid scan pattern are sufficiently diagnostic but in case of uncertainty (for example of uneven distribution of ${ }^{99 \mathrm{~m}} \mathrm{Tc}$ ) the key point of differential diagnosis is the measurement of anti-TSH receptor antibodies (TRAb) $[3,4]$. The value of anti-thyroid peroxidase antibodies (TPOAb) in diagnosing of thyroid disorders is less specific. Although $90 \%$ of patients with Hashimoto's thyroiditis and 70\% with Graves' disease show TPOAb positivity, a moderately increased TPOAb concentration has also been reported in $12-26 \%$ of patients with non-autoimmune thyroid goitre or autonomous thyroid disease and euthyroid adults in the agedependent patterns [5-7]. It is generally believed at the follow-up. Baseline TPOAb values were higher in subjects with coexisting non-thyroid autoimmune disease $(\mathrm{p}=0.041)$. After radioactive iodine therapy, the mean TPOAb level increased in patients with normal baseline TPOAb $(p=0.03)$ and the rates of euthyroidism and hypothyroidism were 61 and 34\%, respectively. The rate of hypothyroidism after radioactive iodine therapy was not significantly different in groups with normal and high baseline TPOAb.

Conclusions: $27 \%$ of patients with non-autoimmune hyperthyroidism were positive for TPOAb. However, baseline TPOAb level did not influence the rate of hypothyroidism at 12 months after radioactive iodine therapy. Our results suggest a more close surveillance after radioactive iodine therapy of patients harboring these antibodies. 
lar goitre (MNTG; $\mathrm{n}=65$ ) and toxic adenoma $(\mathrm{TA} ; \mathrm{n}=35)$ who were referred for RIT to the Department of Nuclear Medicine between January 2010 and March 2011 inclusive. MNTG was defined as a thyroid gland with 2 or more nodules larger than $1 \mathrm{~cm}$, determined by ultrasound and thyroid scan appearance (the presence of foci with increased uptake). Before RIT, 54\% of patients were pre-treated with anti-thyroid drugs, $11 \%$ underwent thyroid surgery and $37 \%$ had recurrent hyperthyroidism. In all patients, clinical indications and eligibility for RIT were individually evaluated by a nuclear medicine specialist. Aside from $\mathrm{NIH}, 9$ patients had a comorbid autoimmune disease, including rheumatoid arthritis, pernicious anaemia, and psoriasis and 15 of them had type 2 diabetes. Subjects with known autoimmune thyroid disease and prior or current malignancy were excluded from the study. Patients were divided into 2 groups according to their baseline TPOAb serum level: with normal (below $60 \mathrm{IU} / \mathrm{ml}$ ) and high TPOAb (above $60 \mathrm{IU} / \mathrm{ml}$ ).

Patients were categorized as euthyroid or as having developed overt hypothyroidism or subclinical hypothyroidism based on clinical and laboratory values for TSH and thyroxine $\left(\mathrm{FT}_{4}\right)$. The euthyroid classification was chosen if the patient's record indicated that $\mathrm{TSH}_{\text {and }} \mathrm{FT}_{4}$ levels were within normal ranges, there were no clinical symptoms associated with altered thyroid status, and there was no thyroid replacement medication use. Overt hypothyroidism was designated if the attending physician diagnosed hypothyroidism and supplemental thyroid hormone was used. Subclinical cases were designated if thyroid replacement therapy could not be confirmed but laboratory test showed elevated $\mathrm{TSH}$ and normal $\mathrm{FT}_{4}$ levels.

\section{Assessment of thyroid function}

Thyroid function tests, including serum $\mathrm{FT}_{4}$ (reference range: $10.0-25.0 \mathrm{pmol} / \mathrm{L}$ ), TSH (reference range: $0.40-4.0 \mathrm{mIU} / \mathrm{L}$ ), TRAb (normal range, below $1.5 \mathrm{IU} / \mathrm{mL}$ ), and TPOAb (normal range: below $60 \mathrm{IU} / \mathrm{mL}$ ), were performed before and 12 months after RIT. TPOAb and $\mathrm{FT}_{4}$ were assessed by radioimmunoassay. TSH was assessed by immunoradiometric assay (BRAHMS $\mathrm{GmbH}$, Germany).

At baseline and 12 months after RIT all the patients underwent thyroid ultrasonography with the use of a $7.5 \mathrm{MHz}$ transducer. A fine needle diagnostic aspiration biopsy was performed in 23 cases to exclude thyroid malignancy prior to RIT.

\section{RIT procedure}

Prior to RIT, all patients underwent a standard assessment of radioiodine uptake after 4,24 and $48 \mathrm{~h}$, as well as a thyroid scan with ${ }^{131} \mathrm{I}$ after $24 \mathrm{~h}$. Palpable structures were directly mapped on the $1: 1$ thyroid scan; $T^{1} 12$ for radioiodine retention was estimated using radioiodine uptake. The volume of thyroid hot nodules was estimated using a formula for spheres or ellipsoids, or directly from their diameters in the ultrasound scan. The entire thyroid volume was estimated by ultrasonography or planimetric method. The radioiodine dose was calculated from the target thyroid volume, planned dose of absorbed radiation, 24-h thyroid ${ }^{131} \mathrm{I}$ uptake, and the effective half-life of ${ }^{131}$ I. Dosimetric data for RIT in the whole group of 100 patients are given in $\odot$ Table 1 .

\section{Statistical analysis}

Descriptive statistics included frequency distributions (number with condition and percentage) for categorical variables and means, standard deviation (SD), and range for continuous variables. To assess changes from baseline to follow-up, we used a paired t-test or nonparametric Mann-Whitney U-test for continuous variables and Chi-square test with Yates' correction for categorical variables. Statistical analyses were performed using Statistica PL package (StatSoft, Poland).

\section{Results}

\section{$\nabla$}

Baseline characteristics of the study population are shown in $\odot$ Table 2. Overall, $65 \%$ of patients had MNTG and palpable neck mass was found in $88 \%$ of them. Recurrence of hyperthyroidism was observed in $37 \%$ of patients. There were no significant differences in TRAb, TSH and $\mathrm{FT}_{4}$ levels between groups with normal and high TPOAb levels. Baseline TPOAb values were significantly higher in subjects with a coexisting non-thyroid autoimmune disease $(\mathrm{p}=0.041)$. Prior to RIT, normal thyroid function was found in 36\% and hyperthyroidism in $64 \%$ of patients. More than $50 \%$ of patients received antithyroid therapy before RIT.

One year after RIT, the rates of euthyroidism and hypothyroidism increased (61 and 34\%, respectively) ( $\odot$ Table 3 ). The prevalence of hypothyroidism was similar in patients with MNTG and TA. Initial thyroid volume, pretreatment with antithyroid drugs, severity of nodular changes/focal lesions in ultrasonography before RIT, total number of recurrences, and radiation dosimetric parameters used for RIT were not associated with the occurrence of hypothyroidism.

Mean values of TPOAb before and after RIT were comparable. However, in the subgroup of patients with normal baseline TPOAb its titres significantly increased after RIT $(p=0.030)$; In subjects with high baseline TPOAb a similar trend was observed but the differences did not reach statistical significance. High TPOAb post RIT was more frequently observed in the group with high baseline titres. Baseline TPOAb did not increase the odds of hypothyroidism after RIT (Odds ratio $=1.09 ; \mathrm{p}=0.473$ ). Mean values of TSH, $\mathrm{FT}_{4}$ and TRAb were comparable in patients with normal and high baseline TPOAb titres.

\section{Discussion}

The concept of this study was based on practical observation that some patients with NIH have an elevated TPOAb level, which may be associated with a higher risk of developing hypothyroidism post RIT $[15,16]$. We found a $34 \%$ prevalence of hypothyroidism at 12 months after RIT which overall is consistent with majority of studies evaluating this therapeutic outcome at a similar time point. High rates of hypothyroidism in NIH treated with RIT were reported both in short- and long-term follow-up. Overall, the rate of hypothyroidism varies from 6 to $55 \%$ within the first several years after RIT [15-18]. Predictably,

\begin{tabular}{ll}
\hline Table 1 & \multicolumn{1}{c}{ Dosimetric data for radioiodine therapy. } \\
\hline Administered ${ }^{131}$ I activity $(\mathrm{mCi})$ & $18.97 \pm 5.63(9.50-32.8)$ \\
\hline Administered ${ }^{131}$ I activity $(\mathrm{MBq})$ & $702 \pm 208(352-1214)$ \\
\hline Thyroid volume $(\mathrm{mL})$ & $28.49 \pm 16.2(7.0-90.0)$ \\
\hline Absorbed dose $(\mathrm{Gy})$ & $250.0(150.0-400.0)$ \\
\hline 24-h uptake of ${ }^{131} \mathrm{I}(\%)$ & $0.35 \pm 0.12(0.15-0.73)$ \\
\hline Effective half-life of ${ }^{131} \mathrm{I}$ (days) & 5.93 \\
\hline & $5.20-6.0$ \\
\hline
\end{tabular}




\begin{tabular}{|c|c|c|c|c|}
\hline Variables & All $(n=100)$ & $\begin{array}{l}\text { TPOAb below } \\
60 \mathrm{IU} / \mathrm{mL} \\
(\mathrm{n}=73)\end{array}$ & $\begin{array}{l}\text { TPOAb above } \\
60 \mathrm{IU} / \mathrm{mL} \\
(\mathrm{n}=27)\end{array}$ & P-value \\
\hline Age (years) & $65.0 \pm 11.9$ & $65.43 \pm 12.5$ & $63.74 \pm 10.5$ & 0.201 \\
\hline Gender (F/M) & $83 / 17$ & $60 / 13$ & $23 / 4$ & 0.235 \\
\hline Multinodular toxic goitre (n) & 65 & $49(67.1 \%)$ & $16(59.3 \%)$ & 0.213 \\
\hline Toxic adenoma $(n)$ & 35 & $24(32.9 \%)$ & $11(40.7 \%)$ & 0.246 \\
\hline Recurrent hyperthyroidism (n) & 37 & $28(38.4 \%)$ & $9(33.3 \%)$ & 0.689 \\
\hline Antithyroid drugs (n) & 54 & $40(54.8 \%)$ & $14(51.9 \%)$ & 0.726 \\
\hline Antithyroid drugs (months) & $49.2 \pm 60.1$ & $42.3 \pm 53.4$ & $55.6 \pm 70.0$ & 0.098 \\
\hline Time of withdrawal of antithyroid drugs (months) & $2.9 \pm 4.5$ & $2.2 \pm 4.4$ & $3.3 \pm 4.9$ & 0.227 \\
\hline Prior thyroid gland surgery $(n)$ & 5 & $4(5.5 \%)$ & $1(3.7 \%)$ & 0.308 \\
\hline Palpable neck mass (n) & 88 & $63(86.3 \%)$ & $25(92.5 \%)$ & 0.402 \\
\hline Hyperthyroidism (n) & 64 & $46(63.0 \%)$ & $18(66.6 \%)$ & 0.787 \\
\hline Normal thyroid function & 36 & $27(36.9 \%)$ & $9(33.3 \%)$ & 0.271 \\
\hline Coexisting autoimmune disease (n) & 9 & $5(6.8 \%)$ & $4(14.8 \%)$ & 0.216 \\
\hline TPOAb (IU/mL) & $271.2 \pm 1059$ & $34.56 \pm 14.2$ & $902.5 \pm 1913$ & 0.001 \\
\hline TRAb (IU/mL) & $0.402 \pm 0.22$ & $0.370 \pm 0.24$ & $0.453 \pm 0.22$ & 0.172 \\
\hline $\mathrm{TSH}(\mathrm{mlU} / \mathrm{L})$ & $0.61 \pm 1.01$ & $0.62 \pm 1.08$ & $0.57 \pm 0.76$ & 0.199 \\
\hline $\mathrm{FT}_{4}(\mathrm{pmol} / \mathrm{L})$ & $15.71 \pm 4.73$ & $15.82 \pm 4.84$ & $15.60 \pm 4.93$ & 0.187 \\
\hline
\end{tabular}

Table 2 Baseline characteristics of the study population.

\begin{tabular}{|c|c|c|c|c|}
\hline \multirow[t]{2}{*}{ Outcomes at 12 months after RIT } & \multirow[t]{2}{*}{ All $(n=100)$} & \multicolumn{2}{|c|}{ Baseline TPOAb } & \multirow[t]{2}{*}{ P-value } \\
\hline & & below $60 \mathrm{IU} / \mathrm{mL}$ & above $60 \mathrm{IU} / \mathrm{mL}$ & \\
\hline Normal thyroid function (n) & $61^{\mathrm{a}}$ & $45(61.6 \%)^{b}$ & $16(59.3 \%)^{b}$ & 0.723 \\
\hline Hyperthyroidism (n) & $5^{c}$ & $4(5.5 \%)^{c}$ & $1(3.7 \%)^{c}$ & 0.245 \\
\hline Hypothyroidism (n) & $34^{c}$ & $22(30.1 \%)^{c}$ & $12(44.4 \%)^{c}$ & 0.050 \\
\hline $\mathrm{TSH}(\mathrm{mIU} / \mathrm{L})$ & $2.82 \pm 1.2^{c}$ & $3.0 \pm 1.27^{c}$ & $2.37 \pm 0.95^{c}$ & 0.876 \\
\hline $\mathrm{FT}_{4}(\mathrm{pmol} / \mathrm{L})$ & $13.61 \pm 4.59$ & $13.56 \pm 4.53$ & $13.67 \pm 4.61$ & 0.917 \\
\hline TRAb (IU/mL) & $0.59 \pm 0.6$ & $0.52 \pm 0.5$ & $0.61 \pm 0.7$ & 0.424 \\
\hline TPOAb (IU/mL) & $455.0 \pm 1250$ & $55.1 \pm 167.7^{\mathrm{a}}$ & $1484.6 \pm 2066$ & 0.001 \\
\hline TPOAb above IU/mL (n) & 32 & 10 & 22 & 0.032 \\
\hline
\end{tabular}

Data are means \pm SD or number (percentage) with condition. $P$ in table refers to comparison between groups with TPOAb below and above $60 \mathrm{IU} / \mathrm{ml}$. ${ }^{\mathrm{P}} \mathrm{P}<0.05$; ${ }^{\mathrm{b}} \mathrm{P}<0.01 ;{ }^{\mathrm{C}} \mathrm{P}<0.001 \mathrm{vs}$. baseline category

it has been suggested that the rate of hypothyroidism may be influenced by a high initial size of the thyroid gland $[9,19]$, prior antithyroid treatment [16], small goiter [9], and a high RAI dose [13]. On the other hand, other studies [17,20-23], as well as our results, failed to confirm such relationships. Additionally, we found that the rate of hypothyroidism after RIT was not associated with the type of NIH (MNTG or TA), recurrences of hyperthyroidism and severity of nodular changes/focal lesions evaluated in ultrasonography.

It has been reported that pre-existing thyroid autoimmunity may predict the development of hypothyroidism after RIT $[13,15,16,22,23]$, although other reports including the present study did not support such association [24]. These discrepancies may be partially related to methodological differences between studies. For example, in studies evaluating the outcomes of RIT in $\mathrm{NIH}$, Carceller et al. [15] included several subjects who were TRAb positive at the time of NIH diagnosis, while in the study of Bolusani et al. [16] $\mathrm{NIH}$ was diagnosed solely by imaging techniques and baseline TRAb was not measured. This may suggest that in these reports a subset of subjects with coexisting Graves' disease were analysed, while the diagnosis of NIH based on scintigraphic appearance alone may be misleading because the scintigraphic 'hot' area may represent healthy background tissue but infiltrated by lymphocytes [25], as for example in Marine-Lenhard syndrome - a rare variant of Graves' disease with coexistent autonomous thyroid nodules $[26,27]$. On the other hand, although we analyzed only the patients who at baseline were negative for TRAb, this does not exclude autoimmune thyroid disease as probably all patients with this condition have a mixture of different TSH receptor antibodies which possess stimulating (TSAb), blocking (TBAb) or neither stimulating nor blocking (thyrotropin binding immunoglobulins) properties [28].

Aside from these methodological considerations related to diagnosis of $\mathrm{NIH}$, baseline TPOAb level has been reported to predict the development of hypothyroidism after RIT, often with some latency and increasing incidence over time $[29,30]$. However, we found that TPOAb was a poor predictor of this condition, similarly as in some other reports [24]. Although we found that the mean TPOAb titer was significantly increased after RIT in the group with its normal baseline level, the rate of hypothyroidism was comparable in patients with normal and high baseline value of this antibody. This may suggest that the occurrence of hypothyroidism might be associated not only with TPOAb performance, but also with RIT-related factors.

The goal of RIT is to destroy thyroid autonomous tissue, which accumulates relatively more radioactive iodine compared to suppressed surrounding tissue. If antithyroid drugs are used prior to ${ }^{131}$ I treatment, the extranodular (suppressed) tissue is metabolically more active and, as a consequence, it may be irradiated by radioiodine. This may lead to undesired damage of healthy thyroid tissue and hypothyroidism $[9,13,24]$. The latter effect may be overlapped by ionizing radiation-induced the 
hibernation-like condition (or prolonged stunning), which temporarily impairs the thyroid function [13,31]. Additionally, it has also been suggested that autonomously functioning micronodules which may accompany toxic adenoma may degenerate and predispose to impaired thyroid functioning post RIT [32].

On the other hand, in euthyroid or subclinical hyperthyroid patients the protective effect of the functional suppression of the extranodular thyroid tissue by the hyperfunctioning toxic adenoma has been observed [17]. This may lead to a lower iodine uptake of the normal thyroid cells at the time of the ${ }^{131}$ I administration.

Thus, the patient outcome after RIT represents a net balance between the amount and quality of residual thyroid parenchyma, which may be influenced by antithyroid drugs, thyroid volume, a coexisting thyroid disease [13], and RIT-induced thyroid autoimmunization caused by the release of thyroid antigens $[14,33]$. Notwithstanding, as suggested by this study, there is a subset of patients with NIH and elevated baseline TPOAb titers (and possibly other thyroid antibodies) in whom RIT potentially may predispose to hypothyroidism. Hence, it could be speculated that patients with clinically or serologically more pronounced autoimmunity react differently to RIT, compared with patients with less pronounced deterioration of the immune system. This might explain the differences in reported outcomes of RIT in autoimmune and non-autoimmune thyroid hyperthyroidism.

In the current study, $9 \%$ of patients had a coexisting non-thyroid autoimmune disease. These patients had a significantly higher TPOAb level in comparison with those without this coexisting condition. To our best knowledge, there have been no previous reports demonstrating a higher prevalence of autoimmune diseases in patients with $\mathrm{NIH}$. However, the association refers rather exclusively to TPOAb (or possibly also other TSH receptor antibodies) than $\mathrm{NIH}$ per se, as autoimmune thyroiditis and Graves' disease are often accompanied by production of organspecific and non-organ-specific antibodies [34,35].

\section{Limitations of the study}

Our study had some limitations. First, due to its observational design, the association between baseline TPOAb and the rate of hypothyroidism at 12 months after RIT may not represent a direct causality, because it may be influenced by several confounding factors known to contribute to the pathogenesis of autoimmune thyroid disease, which were not evaluated in this study. They include serum vitamin D levels, smoking, alcohol consumption, selenium intake, iodine intake, prior infections, parity and estrogen use. Moreover, genetic factors contribute for even $70-80 \%$ to the development of TPOAb and/or TRAb positivity [36]. Therefore, because of the modest sample size and, hence, limited statistical power, we cannot exclude that the differences between patients with high and low baseline TPOAb, which were not adjusted for potential confounders, may be a finding by chance, i.e., a type- 1 error.

Second, we used an arbitrary threshold value of $60 \mathrm{IU} / \mathrm{ml}$ to define the TPOAb positivity. This value refers to the reference range provided by the manufacturer of radioimmunoassay used in our study and, therefore, it may not apply to other assays. Finally, we demonstrated that baseline TPOAb level did not influence the rate of hypothyroidism at 12 months after RIT. However, the overall prevalence of hypothyroidism progressively increase with time from RIT and, hence, it may be widely varied in different time points of evaluation $[16,17]$.
In conclusion, $27 \%$ of patients with NIH were positive for TPOAb. However, baseline TPOAb level did not influence the rate of hypothyroidism at 12 months after RIT. Our results suggest a more close surveillance after RIT of patients harboring these antibodies.

\section{Conflict of interest: None.}

\section{References}

1 Stokkel MP, Handkiewicz Junak D, Lassmann $M$ et al. EANM procedure guidelines for therapy of benign thyroid disease. Eur J Nucl Med Mol Imaging 2010; 37: 2218-2228

2 Bahn RS, Burch HB, Cooper DS et al. Hyperthyroidism and other causes of thyrotoxicosis: management guidelines of the American Thyroid Association and American Association of Clinical Endocrinologists. Thyroid 2011; 21: 593-646

3 Dunkelmann $S$, Wolf $R$, Koch A et al. Incidence of radiation-induced Graves' disease in patients treated with radioiodine for thyroid autonomy before and after introduction of a high-sensitivity TSH receptor antibody assay. Eur J Nucl Med Mol Imaging 2004; 31: 1428-1434

4 Boi F, Piga M, Loy M et al. Hypothyroid Hashimoto's thyroiditis with scintigraphic and echo-color Doppler features mimicking autonomous adenoma. J Endocrinol Invest 2002; 25: 469-472

5 Bjoro T, Holmen J, Krüger 0 et al. Prevalence of thyroid disease, thyroid dysfunction and thyroid peroxidase antibodies in a large, unselected population. The Health Study of Nord-Trondelag (HUNT). Eur J Endocrinol 2000; 143: 639-647

6 Taubner K, Schubert G, Pulzer F et al. Serum concentrations of antithyroid peroxidase and anti-thyroglobulin antibodies in children and adolescents without apparent thyroid disorders. Clin Biochem 2014; 47: 3-7

7 Prummel MF, Wiersinga WM. Thyroid peroxidase autoantibodies in euthyroid subjects. Best Pract Res Clin Endocrinol Metab 2005; 19: $1-15$

8 Bera S, Gupta S, Dutta SK et al. Anti-thyroid peroxidase antibody level in thyroid nodules: with special reference to thyroid neoplasia. Int Res J Pharm 2013; 4: 150-152

9 Le Moli R, Wesche MF, Tiel-Van Buul MM et al. Determinants of longterm outcome of radioiodine therapy of sporadic non-toxic goitre. Clin Endocrinol (Oxf) 1999; 50: 783-789

10 Rizzo M, Rossi RT, Bonaffini $O$ et al. Increased annual frequency of Hashimoto's thyroiditis between years 1988 and 2007 at a cytological unit of Sicily. Ann Endocrinol (Paris) 2010; 71: 525-534

11 Amouzegar A, Bakhtiyari M, Mansournia MA et al. Sex- and age-specific reference values and cutoff points for TPOAb: Tehran Thyroid Study. Thyroid 2016; 26: 458-465

12 McLeod DS, Cooper DS. The incidence and prevalence of thyroid autoimmunity. Endocrine 2012; 42: 252-265

13 Bonnema SJ, Hegedüs L. Radioiodine therapy in benign thyroid diseases: effects, side effects, and factors affecting therapeutic outcome. Endocr Rev 2012; 33: 920-980

14 Pisarek M, Baczyk M, Gryczyńska $M$ et al. Autoimmunization and multinodular large toxic goiter therapy using repeated doses of 131I. Pol Arch Med Wewn 2006; 115: 545-550

15 Boj Carceller D, Liévano Segundo P, Navarro Beltrán P et al. Short-term results of treatment with I-131 in patients with multinodular goiter: effect of the associated degree of hyperthyroidism and other variables. Rev Esp Med Nucl 2011; 30: 156-161

16 Bolusani H, Okosieme OE, Velagapudi $M$ et al. Determinants of longterm outcome after radioiodine therapy for solitary autonomous thyroid nodules. Endocr Pract 2008; 14: 543-549

17 Tzavara I, Tzanela M, Vlassopoulou B et al. Long term thyroid function after 131I treatment for toxic adenoma. Hormones 2002; 1: 99-103

18 Huysmans DA, Corstens FH, Kloppenborg PW. Long term follow up in toxic solitary autonomous thyroid nodules treated with radioactive iodine. J Nucl Med 1991; 32: 27-30

19 Sawicka N, Sowiński J. Correlation between thyroid volume and humoral thyroid autoimmunity after radioiodine therapy in Graves' disease. Endokrynol Pol 2002; 63: 10-13

20 Ahmad AF, Ahmad M, Young ET. Objective estimates of the probability of developing hypothyroidism following radioactive iodine treatment of thyrotoxicosis. Eur J Endocrinol 2002; 146: 767-775

21 Braga $M$, Walpert $N$, Burch $H B$ et al. The effect of methimazole on cure rates after radioiodine treatment for Graves' hyperthyroidism: a randomized clinical trial. Thyroid 2002; 12: 135-139 
22 Andrade VA, Gross JL, Maia AL. The effect of methimazole pretreatment on the efficacy of radioactive iodine therapy in Graves' hyperthyroidism: one-year follow-up of a prospective, randomized study. J Clin Endocrinol Metab 2001; 86: 3488-3493

23 Goldstein R, Hart IR. Follow up of solitary autonomous thyroid nodules treated with 131-I. New Engl J Med 1983; 309: 1473-1476

24 Puille M, Klett R, Steiner D et al. Influence of dose rate on therapeutic success of radioiodine therapy of functional thyroid autonomy. Nucl Med Commun 2005; 26: 727-730

25 Intenzo CM, Capuzzi DM, Jabbour S et al. Scintigraphic features of autoimmune thyroiditis. Radiographics 2001; 21: 957-964

26 Cakir M. Marine-Lenhart syndrome. J Natl Med Assoc 2005; 97: 1036-1038

27 Ruggeri RM, Campenni A, Sindoni A et al. Association of autonomously functioning thyroid nodules with Hashimoto's thyroiditis: study on a large series of patients. Exp Clin Endocrinol Diabetes 2011; 119: 621-627

28 DeGroot LJ. Graves' Disease and the manifestations of thyrotoxicosis. [Updated 2015 Jul 11]. In: De Groot LJ, Beck-Peccoz P, Chrousos G et al. (eds.). Endotext [Internet]. South Dartmouth (MA): MDText. com, Inc.; 2000 Available from http://www.ncbi.nlm.nih.gov/books/ NBK285567/

29 Nygaard B, Faber J, Veje A et al. Transition of nodular toxic goiter to autoimmune hyperthyroidism triggered by 131I therapy. Thyroid 1999; 9: 477-441
30 Custro N, Ganci A, Scafidi V. Relapses of hyperthyroidism in patients treated with radioiodine for nodular toxic goiter: relation to thyroid autoimmunity. J Endocrinol Invest 2003; 26: 106-110

31 Sheehan MT, Doi SAR. Transient hypothyroidism after radioiodine for Graves' disease: challenges in interpreting thyroid function tests. Clin Med Res 2016; 14: 40-45

32 Freitas JE. Therapeutic options in the management of toxic and nontoxic goiter. Semin Nucl Med 2000; 30: 88-97

33 Laurberg $P$, Wallin $G$, Tallstedt $L$ et al. TSH-receptor autoimmunity in Graves' disease after therapy with anti-thyroid drugs, surgery, or radioiodine: a 5-year prospective randomized study. Eur J Endocrinol 2008; 158: 69-75

34 Lazúrová I, Benhatchi K, Rovenský J et al. Autoimmune thyroid disease and autoimmune rheumatic disorders: a two-sided analysis. Ann NY Acad Sci 2009; 1173: 211-216

35 Al-Awadhi AM, Olusi S, Hasan EA et al. Frequency of abnormal thyroid function tests in Kuwaiti Arabs with autoimmune diseases. Med Princ Pract 2008; 17: 61-65

36 Wiersinga WM. Clinical relevance of environmental factors in the pathogenesis of autoimmune thyroid disease. Endocrinol Metab (Seoul) 2016; 31: 213-222 